\title{
Resource-rational approach to meta-control problems across the lifespan
}

\author{
Alexa Ruel ${ }^{1}$, Sean Devine ${ }^{2} \&$ Ben Eppinger ${ }^{1,3,4}$ \\ ${ }^{1}$ Department of Psychology, Concordia University, Montreal, Canada \\ ${ }^{2}$ Department of Psychology, McGill University, Montreal, Canada \\ ${ }^{3}$ Faculty of Psychology, Technische Universität Dresden, Dresden, Germany \\ ${ }^{4}$ PERFORM Center, Concordia University, Montreal, Canada
}

Running head: Meta-control across the lifespan

Corresponding author: alexa.ruel@mail.concordia.ca

Keywords: meta-control, cognitive control, decision-making, children, older adults

Conflict of Interest: The authors declare no conflict of interest.

Acknowledgements: The authors thank Rasmus Bruckner for his comments on the manuscript.

Funding Information: This work was supported by a grant from the German Research Foundation (DFG) [SFB 940/2 B7] as well as the Natural Sciences and Engineering Research Council of Canada (NSERC), [funding reference number N01882]. Furthermore, this research was undertaken, in part, from the Canada Research Chairs program. 
Meta-control across the lifespan

\begin{abstract}
Over the last decade, research on cognitive control and decision-making has revealed that individuals weigh the costs and benefits of engaging in or refraining from control and that whether and how they engage in these cost-benefit analyses may change across development and during healthy aging. In the present article, we examine how lifespan age differences in cognitive abilities affect the meta-control of behavioral strategies across the lifespan and how motivation affects these trade-offs. Based on accumulated evidence, we highlight two hypotheses that may explain the existing results better than current models. In contrast to previous theoretical accounts, we assume that age differences in the engagement in cost-benefit trade-offs reflect a resource-rational adaptation to internal and external constraints that arise across the lifespan.
\end{abstract}


Meta-control across the lifespan

When trying to solve a complex goal-directed task such as navigating to a restaurant in a new city, we can apply different types of strategies (or combinations of strategies). We can learn the layout of the new city from a map and then use this knowledge to reach the restaurant, or we can simply put the address in our GPS and let it guide us there. For we can engage in either strategy or a combination of both, we are left with a meta-control problem: we have to decide which strategy (or combination thereof) to engage in based on the expected outcomes and the effort each one requires. In the example above, most people would engage in the simple and less effortful strategy of using a navigation device. However, there are scenarios in which we might have a long-term interest in developing an internal representation of a cognitive map of the city (e.g. becoming a resident of the city) and might therefore decide to engage in the more effortful strategy. In these cases, one must balance the immediate costs of engaging in effortful activity (the time spent committing the city layout to memory) against the benefits such activity confers (a more flexible and rapid navigation in the future).

In this manuscript, we examine how individuals of different ages solve the cost-benefit trade-offs that may arise when different behavioral strategies are available to perform a given task. We assume that constraints in cognitive capacity (as commonly observed in children and older adults) are one important determinant of how such trade-offs are solved. We start by describing how cost benefit trade-offs are studied using cognitive control and decision-making paradigms and examine the performance of younger adults in these tasks. We then consider what is known about developmental and aging effects on the engagement in these trade-offs.

Specifically, we review how age-related differences in cognitive abilities affect the meta-control of behavioral strategies across the lifespan. Finally, we consider how motivational factors such as incentives may shift these cost-benefit analyses in different age groups. We conclude by 
Meta-control across the lifespan

presenting two hypotheses to explain age differences in the meta-control of behavioral strategies. These theoretical accounts assume that age differences in the engagement in cost-benefit tradeoffs reflect a resource-rational adaptation to internal and external constraints in children and older adults.

\section{Trade-offs in Cognitive Control}

Cognitive control requires the encoding and maintenance of task representations and has been suggested to rely on a set of basic cognitive abilities, consisting of both working and episodic memory as well as attentional functions (Cattell, 1987; Salthouse, 1990; Willis \& Marsiske, 1991). Many activities of daily life (such as navigating to a specific restaurant in a new city, to return to an earlier example) require the efficient engagement and maintenance of such control to achieve one's goal. Yet, sustained engagement in cognitive control (i.e., continuously engaging in cognitive effort towards a goal) is effortful, and therefore, similarly to physical effort, tends to be aversive (Botvinick, 2007; Botvinick \& Braver, 2015; Kool, McGuire, Rosen \& Botvinick, 2010; Kool Shenhav \& Botvinick, 2017). As a consequence, we often choose to refrain from engaging in cognitive control and instead engage in simpler, habitual, strategies that may be suboptimal in terms of performance, but are cognitively cheaper to perform (Botvinick, 2007; Botvinick \& Braver, 2015; Kool et al., 2010; Kool et al., 2017).

Therefore, how we solve this meta-control problem depends on how much value we attribute to the outcome of a chosen strategy as well as the cost that it entails. For example, take a classic variant of the Ericksen flanker task (Ericksen \& Ericksen, 1974) in which participants must decide if a central stimulus is pointing left or right. Flanking this center stimulus, is a set of similar stimuli that either point in the same direction as the center stimulus (congruent) or opposite to it (incongruent). The difficulty of the incongruent trials is evidenced by behavioral 
Meta-control across the lifespan

deficits such as reduced accuracy and slower reaction times (Ericksen \& Ericksen, 1974).

Throughout the task then, participants must engage in cognitive control to monitor conflict and to inhibit incongruent information when it arises. To incentivize maximal engagement in such control, variants of the Flanker task provide a small monetary incentive for each correct response participants make (see Hsieh, Li \& Tsai, 2010). In other variants, rewards fluctuate across trials (Devine, Otto, Bolenz, Reiter \& Eppinger, 2019; see Figure 1A). In these cases, participants are presented with a meta-control decision: either to invest resources into sustained control to maximize performance and reward, or to reduce control and risk foregoing potential gains. To resolve this decision, individuals typically engage in a cost-benefit analysis, investing more control when its costs are offset by higher rewards. Alternatively, participants may decide that the value of the reward is (subjectively) insufficient to offset the costs associated with cognitive control and instead default to simple, less cognitively demanding control strategies. Similar effort-based control tasks have been employed to show that individuals engage in these costbenefit analyses regarding the choice to engage in cognitive control. These include Stroop-like attention tasks (Padama \& Pesoa, 2011), free recall memory tasks (Libby \& Lipe, 1992), and stop-signal tasks (Leotti \& Wagner, 2010) among others.

\section{Trade-offs in Decision-Making}

Such meta-control problems are not specific to cognitive control processes. They are also encountered in the context of learning or decision-making. In many real-world situations, we can engage in two choice strategies of different complexities. The first is consists of simple habitual strategies that rely on previous experiences of similar decisions. The second refers to elaborate goal-directed strategies that involve forward planning. In the reinforcement learning (RL) literature, these strategies are referred to as model-free (MF) and model-based (MB) learning, 
Meta-control across the lifespan

respectively. MF decision-making is computationally inexpensive as it relies on learned actionoutcome associations whereas MB learning is computationally expensive as it involves the mental simulation of candidate actions based on a learned internal model of the task (see Daw, Gershman, Seymour, Dayan \& Dolan, 2011). When faced with the choice to engage in different decision-making strategies, individuals weigh the cognitive effort required for each strategy against the expected value of the decision outcome and prioritize the action that maximizes reward. Furthermore, depending on the rate of change (volatility) of the environment, the optimality of this choice may be subject to re-evaluation over the course of the task.

Consequently, it has been proposed that the arbitration between MB and MF strategies is achieved by integrating the costs and benefits of each strategy, similar to the analysis of costs and benefits associated with engaging in cognitive control (Kool et al., 2017; Kool, Cushman \& Gershman 2018).

In their recent work Kool and colleagues (2018) have tried to capture such arbitrations using a variant of a two-stage Markov decision task (see Daw et al., 2011 and Figure 1B). In this task participants can either engage in a model-free strategy that relies on learned action-reward associations or they can adopt a model-based strategy that also accounts for the probabilistic transition structure that connects the two stages of the task. Critically, in their variant of the paradigm, reward cues of different magnitudes are provided at the beginning of each trial to induce cost-benefit trade-offs (see Figure 1B). The results of this study show that participants demonstrate greater MB decision-making on trials with larger compared to smaller reward incentives. In other words, subjects engaged in cost-benefit trade-offs when making meta-control decisions about resource investment, such that they only engaged in MB control when the benefits of doing so outweighed the costs. Similarly, Kool et al., (2018) assessed whether people 
adjust their degree of MB decision-making as the complexity of required planning was

manipulated. Across three experiments, they found that the allocation of MB decision-making was proportional to the degree of planning demands associated with it.

\section{A) Flanker Task}

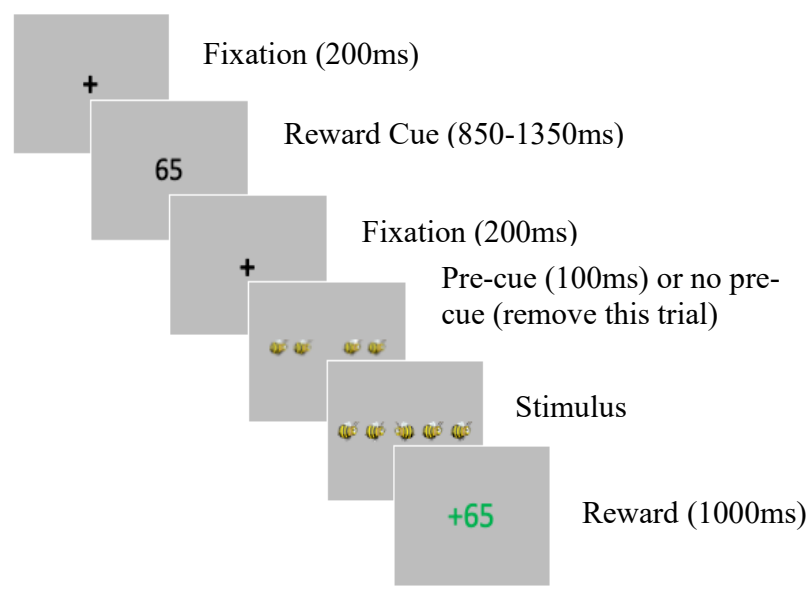

B) Two-stage decision-making task

i)

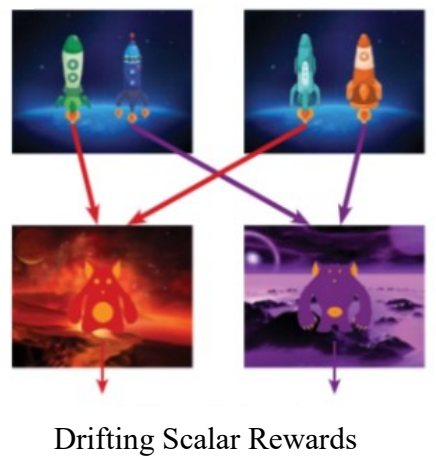

ii)

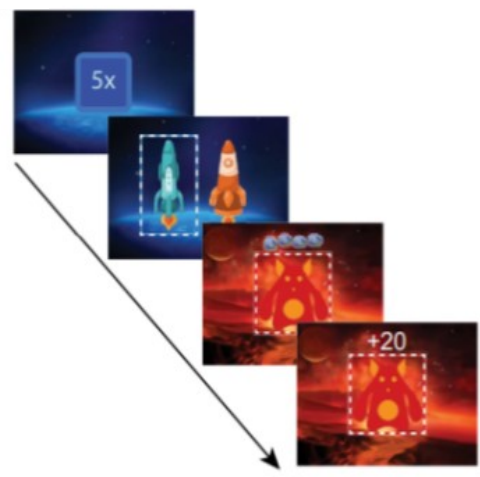

Fig 1. A Schematic figure of the Flanker task. In this paradigm, participants have to indicate whether the center bee is flying to the left or right. On congruent trials, the flanking bees are flying in the same direction. On incongruent trials, the flanking bees fly in the opposite direction. Before each trial, a reward cue which indicates how many points can be obtained if the correct response is given is shown on the screen. (Adapted from "Cognitive Resource Limitations shift effort trade-offs across the lifespan" by Devine, S. T., Otto, R., Bolenz, F., Reiter, A. M., \& Eppinger, B. (2019)). B Schematic figure of the modified two-stage decision-making task. i) Each trial begins with one of two possible states in which participants must decide between different pairs of spaceships which allows them to transition to either of two second stage states (i.e., a red vs. purple planet). The relationship between spaceships and planets is deterministic. Each planet hosts a single alien delivering fluctuating rewards which slowly changes on a trialby-trial basis. ii) At the start of each trial, participants are presented with a reward multiplier indicating if points on that trial would be multiplied by 1 (low stake trials) or 5 (high stake trials). Reprinted from "Cost-Benefit Arbitration Between Multiple Reinforcement-Learning Systems” by Kool, W., Gershman, S.J. \& Cushman, F.A. (2017).

\section{Cognitive Control and Decision-Making: Two Sides of the Same Coin}


Meta-control across the lifespan

In the last two sections, we discussed how individuals engage in cost-benefit trade-offs when engaging in meta-control. Despite the apparent differences between the two, our decision to engage in a more or less effortful behavioral strategy seems to be governed by a similar strategy: namely, an effort-reward cost-benefit trade-off. How can this relationship be understood from a theoretical perspective? Several models have been proposed that summarize and elucidate decades of research on motivation and control.

\section{Theoretical Models explaining the Relationship between Motivation and Cognitive Control}

\section{Reward-based Models}

One of the leading theoretical approaches explaining the relationship between motivation and control focuses on the theme of reward maximization. Reward-based models frame control as involving a series of decisions made with the aim of maximizing utility. These decisions weigh both the anticipated rewards as well as the expected costs of engaging in such effort; resulting in a cost-benefit analysis in which the value of the reward is discounted by the amount of cognitive effort that needs to be exerted. Dixon and Christoff (2012) support this hypothesis, and demonstrate that participants rarely decide to engage in cognitive control when the outcome of doing so is equal or less than the reward given for choosing the task that requires no cognitive effort. Yet, participants frequently choose to invest resources into control when it is expected to result in a large reward.

Expected Value of Control Model. A closely related reward-based model, the expected value of control model (EVC model), integrates the expected reward, the amount of control required to obtain the reward, and the cost in terms of cognitive effort (Shenhav, Botvinick \& 
Meta-control across the lifespan

Cohen, 2013). The cumulative reward expected after engaging in control with a particular intensity is subtracted from the cost of the exertion itself. These cost-benefit analyses guide decisions which are made in order to optimize EVC. Work by Kool and Botvinick (2014) support this model by demonstrating that decisions to engage in cognitive control come from weighing both income and leisure (i.e., lack of cognitive effort). Specifically, they find that wage reductions lead to a decrease in cognitive effort to settle for a smaller reward, while wage increases lead participants to give up leisure - engaging in more effort to obtain the promised reward.

\section{Opportunity Cost of Time}

Another account incorporates a closely related cost: time. The opportunity cost of time holds that the cost of effort also depends on the rewards that are being foregone in order to obtain the current (anticipated) outcome. That is, because the brain has limited resources, we must not only choose to engage in control based on immediate reward, but also based on what anticipated actions and rewards will be foregone by engaging in control now instead of later (Kurzban, Duckworth, Kable \& Myers, 2013). Thus, in contrast to the previous models, the opportunity cost of time also considers when it is most beneficial for a participant to engage in cognitive control based on remaining resources as well as the costs and benefits of doing so. Also grounded in RL, this model defines the trade-off as the weighting of two costs. First is the effort assumed necessary to produce faster actions and second is the opportunity cost inherent in acting more slowly, in which case there is a delay in getting to the next rewards. In other words, the opportunity cost of time is the average reward rate per unit time (Bierholm, Guitart-Masip, Economides, Chowdhury, Düzel, Dolan \& Dayan, 2013; Niv, Daw, Joel \& Dayan, 2007). A recent study by Otto and Daw (2019) leveraged recent theories on the opportunity cost of time to 
Meta-control across the lifespan

examine how manipulating the average reward rate affects young adults' decisions to engage in cognitive effort. They found that subjects tuned their level of effort to the average reward rate. When the opportunity cost of time was high, participants made more errors and responded more quickly as compared to when the opportunity cost of time was lower. Due to the perceptual nature of the decision-making task, responding faster meant that participants accumulated less evidence before making a response which resulted in reduced accuracy, but also led to a reduction in the cost associated with accumulating more evidence. This pattern of behavior is consistent with the conclusion that when the opportunity cost of time is high, participants withdraw cognitive effort.

Uniting these theories is a set of explicit assumptions (see Box 1). However, an implicit assumption of these models is that they consider value and costs to be stable across participants. That is, so far in this paper, we have treated effort as a variable that is determined by the characteristics of the task, while ignoring important task-independent individual differences that affect effort investment. For instance, structural and/or functional limitations in brain function may reduce ones' ability to engage in cognitive control or in a MB decision strategy. This should lead to steeper discounting functions; whereby expected rewards will be more heavily adjusted for by increased processing costs. In what follows we will consider how age-related cognitive limitations affect such meta-control processes across the lifespan. 
Box 1.

Formalizing theories of cost-benefit trade-offs

The models presented above share a common set of assumptions, based in computational theories of RL:

1. The brain has limited computational resources that can be invested towards a task at any given time

2. Investing these resources at one time point depletes them for later use

3. The investment of these resources is dictated by an optimization function that aims to maximize rewards and minimize costs

4. Control is costly, but can yield high rewards

While these models share these basic assumptions, they differ in how they formalize them. The reward-based model (Dixon \& Christoff, 2012) suggests the following:

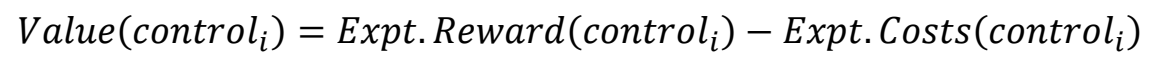

where the value of engaging in cognitive control at time $i$ is proportional to the rewards expected from doing so, adjusted for the costs inherent to mobilizing the necessary resources.

The EVC model makes a similar claim but is probabilistic and summative in nature.

$$
\begin{aligned}
& \text { EVC (signal, state }) \\
& \left.\qquad=\left[\sum_{i} p\left(\text { outcome }_{i} \mid \text { signal }_{i} \text { state }_{i}\right) \text { Value }_{\left(\text {outcome }_{i}\right)}\right)\right]-\operatorname{Cost}(\text { signal })^{-}
\end{aligned}
$$

where the expected value one could obtain from engaging in control is a function of the probability of obtaining reward if resources are mobilized, adjusted for the costs of doing so. Thus, control is only engaged in when EVC is positive; that is, in all probability, the benefits outweigh the costs.

Finally, opportunity cost models posit that control is invested in accordance with the average reward per unit time, which can be formalized as follows:

$$
\dot{r}_{i+1}=(1-\alpha)^{\tau}+\dot{r}_{i}+\left(1-(1-\alpha)^{\tau}\right) \frac{R_{i}}{\tau}
$$

Here, the average reward per unit time is updated each time point $i$ based on $\tau$, the time since the last update, which depends on participants engagement and is reflected in response time, and $\alpha$, a free learning rate parameter. $R$ here represents the reward magnitude at time point $i$. 
Meta-control across the lifespan

\section{Cost-Benefit Trade-offs Across the Lifespan}

Inverse U-shaped developmental curves have been found for several basic cognitive processes such as attention, memory and inhibition (see Kail \& Salthouse, 1994; Li et al., 2013). That is, just as basic cognitive processes have been shown to improve from childhood to adulthood, they have been shown to follow the opposite pattern, decreasing with increasing age. Thus, as we age, the amount of cognitive control an individual can successfully harness will vary at the individual level but more importantly according to age-related changes in cognitive resources.

Cognitive control and MB decision-making performance seems to peak in young adults. Moreover, behavioral control is bolstered even further when young adults are provided with greater incentives (e.g., money) for higher performance (Kool et al, 2017, 2018; Padmala \& Pessoa, 2011). In line with the theories presented in the last section, it seems that incentives serve as an additional benefit in young adults' cost-benefit analysis, making it worthwhile for them to engage in greater control. In the domain of decision-making, consistent with the outcome of a cost-benefit analysis, participants demonstrate more MB decision-making on trials with larger incentives, but only when it leads to better performance on the task (i.e., greater benefits, often meaning more money) (Kool et al., 2017).

In contrast to younger adults, children and older adults likely compute these cost-benefit analyses differently due, in part, to their reduced cognitive abilities.

\section{Children.}

To study effort reward trade-offs in children, Chavalier (2017) asked children to perform a Cognitive Effort Discounting paradigm which allowed for the estimation of how much reward 
Meta-control across the lifespan

children were willing to forgo in order to conserve cognitive effort. Children aged 7 to 12 years avoided harder tasks, not due to their lower likelihood of success or motivation, but due to the additional effort the task required. Specifically, children at this age already require significant incentive to perform more difficult N-back tasks (e.g. 2-back vs. 1-back) (Chevalier, 2018). Using a demand selection task (DST) (see Kool et al., 2010), in which participants must make a recurring choice between two tasks that each varied in cognitive demands, findings by Niebaum et al., (2019) echo those by Chevalier (2018). Specifically, Niebaum and colleagues (2019) show that 11- to 12-year old's, like adults, exhibit a significant preference for selecting the less demanding task. In contrast, younger children (6- to 7-year old's) do so only when provided with feedback and explicitly instructed to select the easier task. In line with these findings, when provided trial-by-trial feedback and familiarization with each task before making a choice, 5year old children have been shown to demonstrate the slight ability to discriminate between task difficulties (O’Leary \& Sloutsky, 2017). Similarly, when younger children (i.e., 5- to 7- year old's old) were encouraged to monitor their performance by estimating their own feedback, they performed better on a flanker task than children who received no feedback as well as better than children who received standard feedback (Hadley, Acluche \& Chevalier, 2019). These results suggest that metacognitive reflection may be a key for efficient cognitive control engagement. Together, it seems that adaptive meta-control of behavioral strategies emerges with development between the ages of 10 and 13 years. Interestingly, this is consistent with the developmental trajectory of brain areas involved in the ability to flexibly integrate learned associations between stimuli, events and contexts (i.e., prefrontal cortex or PFC)(Menon, Boyett-Anderson, \& Reiss, 2005; Ofen, Chai, Schuil, Whitfield-Gabrieli \& Gabrieli, 2012; Shohamy \& Turk-Browne, 2013; Zeithamova, Schlichting \& Preston, 2012), the implementation of cost-benefit analyses (i.e., 
Meta-control across the lifespan

dorsolateral anterior cingulate cortex or dACC)(Shenhav et al., 2013) and the avoidance of cognitively demanding tasks (i.e., connectivity between PFC and dACC (Shenhav, Musslick, Lieder, Kool, Griffiths, Cohen, \& Botvinick, 2017; Shenhav et al., 2013).

As children develop the ability to adapt to demands in control via cost-benefit trade-offs they also start to increase cognitive effort if the incentive motivates them enough to respond. For instance, Strang and Pollack (2014) found that children (9- to 11-years old), adolescents (14- to 16-years old), and adults all demonstrated a shift to greater cognitive control when provided with greater rewards. In contrast, findings by Insel and colleagues (2017) revealed that participants aged between 13-18 performed similarly across high and low value trials on a go/no-go task. Nevertheless, participants who demonstrated an enhanced performance on high-stake trials showed greater connectivity between the ventral striatum and the VLPFC (ventrolateral prefrontal cortex) which has been implicated in the maturation of cognitive control, RL and value-based decision-making (Insel, Kastman, Glwnn \& Somerville, 2017). Together, their findings suggest that adolescents show clear neural evidence of the engagement in cognitive control yet continue to show improvements as they mature. Even children as young as five have recently been shown to demonstrate greater MB learning, but only on an age-appropriate task in which MB decision-making outperformed MF learning (Smid, Kool, Hauser \& Steinbeis, 2020). Yet, incentivizing more complex decision-making tasks reveals that with increasing age, adolescents show greater adaptation of model-based decision making to outcome magnitudes (Bolenz \& Eppinger 2020). Thus, it seems that meta-control continues to develop into young adulthood.

Overall, it seems that the sensitivity and ability to adapt to cognitive control and demands on MB learning emerge with development, allowing children as young as 11-12 years old to 
Meta-control across the lifespan

engage in adult-like cost-benefit trade-offs. Nevertheless, due to sharp differences in findings using different tasks (e.g. cognitive control tasks versus decision-making tasks), these developmental changes may also be task dependent.

\section{Older Adults.}

A considerable body of work demonstrates that cognitive control declines in aging adults (Mayr, Speiler \& Kliegl, 2001; McDowd \& Shaw, 2000). In a conflict monitoring task, older adults demonstrate greater difficulties in cognitive control showing greater conflict cost than younger adults (Li, Hämmerer, Müller, Hommel \& Lindenberger, 2009). Similarly, in task switching experiments, older adults tend to show larger interference effects than younger adults (Eppinger, Kray, Mecklinger \& John, 2007) and demonstrate significant difficulties selecting between task sets (Kray, Eber \& Lindenberger, 2004). Further, subjective perceptions of task difficulty vary on an individual level and seem to incorporate aspects of task demands as well as experience of their own efforts during the completion of the task, increasing the perceived demands of cognitive control (Westbrook, Kester, \& Braver, 2013). Consequently, older adults typically err on the side of caution, engaging in habitual responses instead of cognitive control or MB decision making following a cost-benefit analysis (Hess, Smith, \& Sharifian, 2016; Westbrook et al., 2013).

Nevertheless, recent work has shown that for specific tasks, the excessive cautiousness with which older adults approach cost-benefit trade-offs can be mitigated by motivational incentives, leading to an upregulation of control. For instance, older adults can attain motivationrelated performance enhancements in tasks that require cognitive control. Although their responses slow down, they seem to do so to maintain accuracy, allowing them to engage in more 
Meta-control across the lifespan

cognitive control (Starns \& Ratcliff, 2010; Yee, Adams, Beck \& Braver, 2019). In contrast to cognitive control, motivational incentives do not seem to lead to increased MB decision-making in older adults, even when engaging in this more complex strategy leads to better task outcomes (Bolenz, Kool, Reiter \& Eppinger, 2019; Patzelt, Kool \& Gershman, 2020). Thus, it seems that the reduced MB learning seen in older adults cannot be explained by a reduced willingness to engage in this strategy, but rather is due to age-related limitations in the cognitive processes involved in $\mathrm{MB}$ decision-making. In line with this hypothesis are recent findings demonstrating that older adults do demonstrate increased MB control when the task demands on representing the decision-making structure are reduced (Ruel, Bolenz, Li \& Eppinger, in prep).

Overall, it seems that older adults may be aware of their cognitive limitations, taking these into consideration when engaging in meta-control and its cost-benefit trade-offs. When sufficiently incentivized, older adults demonstrate increased performance on basic cognitive tasks, yet do not show greater meta-control when faced with a decision-making task. One possible explanation for these findings is that the older adults recognize their inability to engage in greater control in decision-making tasks (i.e., a high and unsurmountable cost), failing to represent the task structure, and thus resort to a habitual strategy (Bolenz et al., 2019). However, administering a simpler cognitive task might determine that older adults are capable and sufficiently enticed to engage in a greater amount of cognitive control. While it is unclear if children may also recognize their cognitive limitations, they demonstrate a similar effect whereby under the age of 11 or 12 years, children fail to adapt to engage in cost-benefit tradeoffs. Ultimately, , when task demands are adjusted to complement cognitive abilities, participants in both age groups successfully engage in trade-offs and even show enhanced metacontrol in response to motivational in incentives (Ruel et al., in prep; Smid et al., 2020). 


\section{Resource-rational theories of meta-control across the lifespan}

Several theoretical models have been proposed to explain the computations by which individuals weigh the costs and benefits of engaging in control, and how motivation may influence the resulting trade-off (see Theoretical Models explaining the Relationship between Motivation and Cognitive Control). However, these models fail to consider the impact of lifespan changes in cognitive limitations of the ability to engage in meta-control or explain these changes in meta-control as deficit-driven (Dixon \& Christoff, 2012; Shenhav et al., 2013).

Griffiths (Griffiths et al., 2015; Leider \& Griffiths 2020; Shenhav et al., 2017) has proposed a theory that integrates principles of the rational use of limited resources principles with realistic cognitive constraints. Based on foundational work by Herbert Simon $(1955 ; 1956)$ who argued that rational decision strategies must be adapted to both the structure of the environment and the mind's cognitive limitations, Griffith's resource-rational model considers which cognitive operations are possible for individuals to complete and how costly those operations are in terms of time and available resources. In contrast to models reviewed above, the resource-rational model considers the cognitive resources available to the individual. These models posit that people can make (boundedly) rational decisions based on characteristics of the task, the environment, and — most importantly — the individual. We argue that such a model better accounts for current empirical findings of individuals' meta-control abilities across the lifespan.

In what follows, we review two novel theoretical accounts that may jointly explain how cost-benefit analyses change across the lifespan. In line with a resource-rational model, rather than suggesting that children and older adults are sub-optimal in their meta-control, these two 
Meta-control across the lifespan

models hold that they are boundedly optimal decision-makers within the constraints of their limited cognitive abilities.

The first model has been proposed in the context of a study examining performance of children, young adults, and older adults in a predictive-inference task in which participants had to continuously update their predictions based on uncertain (noisy) information. Specifically, participants had to adjust their predictions regarding the position of an invisible helicopter based on bags dropped by the helicopter that provided uncertain information about the true hidden location (Bruckner, Nassar, Li \& Eppinger, 2020; Nassar, Bruckner, Gold, Li, Heekeren \& Eppinger, 2016). One major empirical finding by Bruckner and colleagues (2020) was that children and older adults showed strongly enhanced perseverative behavior when compared to younger adults. That is, they failed to update their predictions and this effect was particularly pronounced for small prediction errors, when the update is potentially more effortful. The authors show that perseverative behavior in children and older adults can be explained using a satisficing model in which children and older adults stop updating their predictions at a threshold that they are satisfied with rather than continuing to update their predictions optimally. 
A)

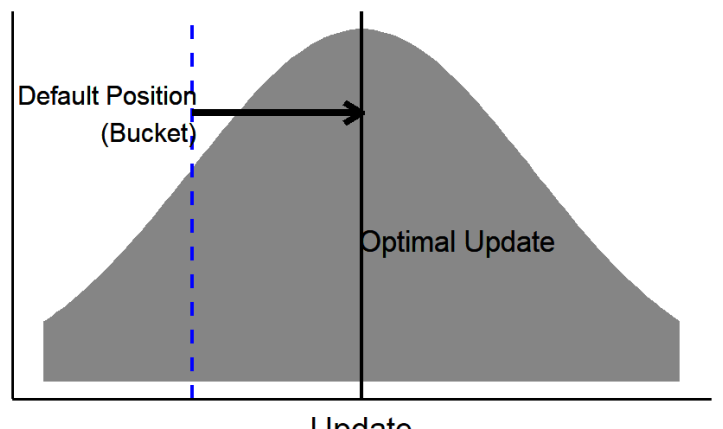

Update

B)

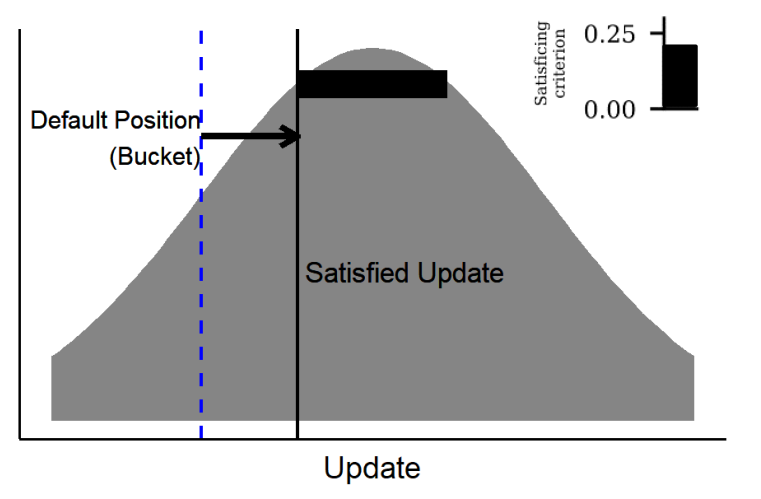

C)

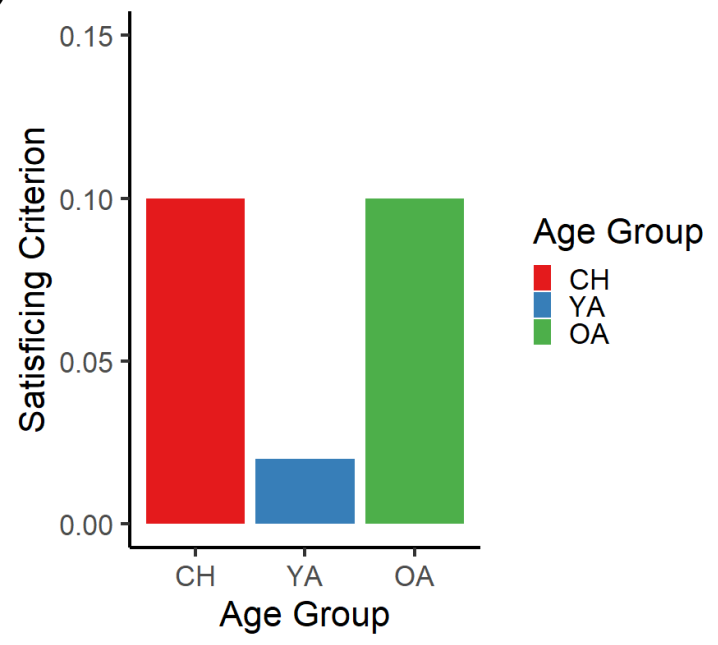

Fig 2. Visual representation of the satisficing model. A The model predicts that normative behavior (similar to younger adults) is characterized by updates of predictions to the most likely outcome (mean of the distribution). B In contrast, in line with a satisficing model, children and older adults update their predictions until reaching the satisficing threshold, at which time an acceptable position is established. C Example satisficing criteria by age group.

In the context of more standard choice tasks, the satisficing model would suggest that children and older adults may stop adjusting their behavior because they do not see the additional value, and/or because they do not have the cognitive control abilities to do so. In line with the studies reviewed above, the satisficing model predicts that children and older adults do not engage in greater cognitive control when small behavioral adjustments are needed as the costs associated with doing so does not exceed the associated benefits (Bolenz et al., 2019; Decker, Otto, Daw \& Hartley, 2016). Yet, when provided with greater incentives or when task demands 
Meta-control across the lifespan

are reduced, leading the benefits to now outweigh the costs, both children and older adults may demonstrate greater control (Ruel et al., in prep; Smid et al., 2020). Interestingly, this hypothesis is in line with Simon's suggestion that the pressure for adaptation makes it rational for children and older adults to use a heuristic that selects the first option that is good enough instead of trying to find the ideal option (Simon, 1955; 1956). Simply put, perseveration might occur because the cognitive demands of the task exceeding the individual's cognitive resources (see Gershman, 2020).

Another theoretical explanation comes from recent findings examining the opportunity cost of time across the lifespan. By using paradigms in which the average reward rate per unit time fluctuates over the course of the task, these studies allow for the examination of participants' decisions to engage in cognitive control (i.e., meta-control) as reward rates change. This work demonstrated that while children and older adults expend cognitive effort when the opportunity cost of time is high (i.e., when the average reward that can be obtained by answering correctly per unit time is high), younger adults and adolescents withhold effort (Devine et al., 2020). The authors suggest that these differences in effort avoidance might be explained from a resource-rational perspective. Specifically, they argue that young adults and adolescents may withhold resources when rewards are abundant (i.e., opportunity costs are high), preferring instead to save their finite cognitive resources for when rewards are infrequent. Instead, children and older adults, recognizing their limitations, may prefer to invest resources when it yields maximum rewards, without considering the future depletion of resources. Extrapolating from these findings, we suggest that children and older adults may have a sweet spot at which the incentivization structure and the cognitive demands of the task are tailored to the needs of the different age groups (see Devine et al., 2019; Devine, Neumann, Otto, Bolenz, Reiter \& 
Meta-control across the lifespan

Eppinger, 2020). That is, based on a combination of age-related and individual cognitive limitations, we suggest that each participant will have a "sweet spot" at which they will demonstrate optimal engagement in meta-control. Critically, this sweet spot will reflect individuals' age-related cognitive limitations, while also maintaining that their decisions are rational and optimal given the boundaries of the task, the environment, and their cognitive abilities.

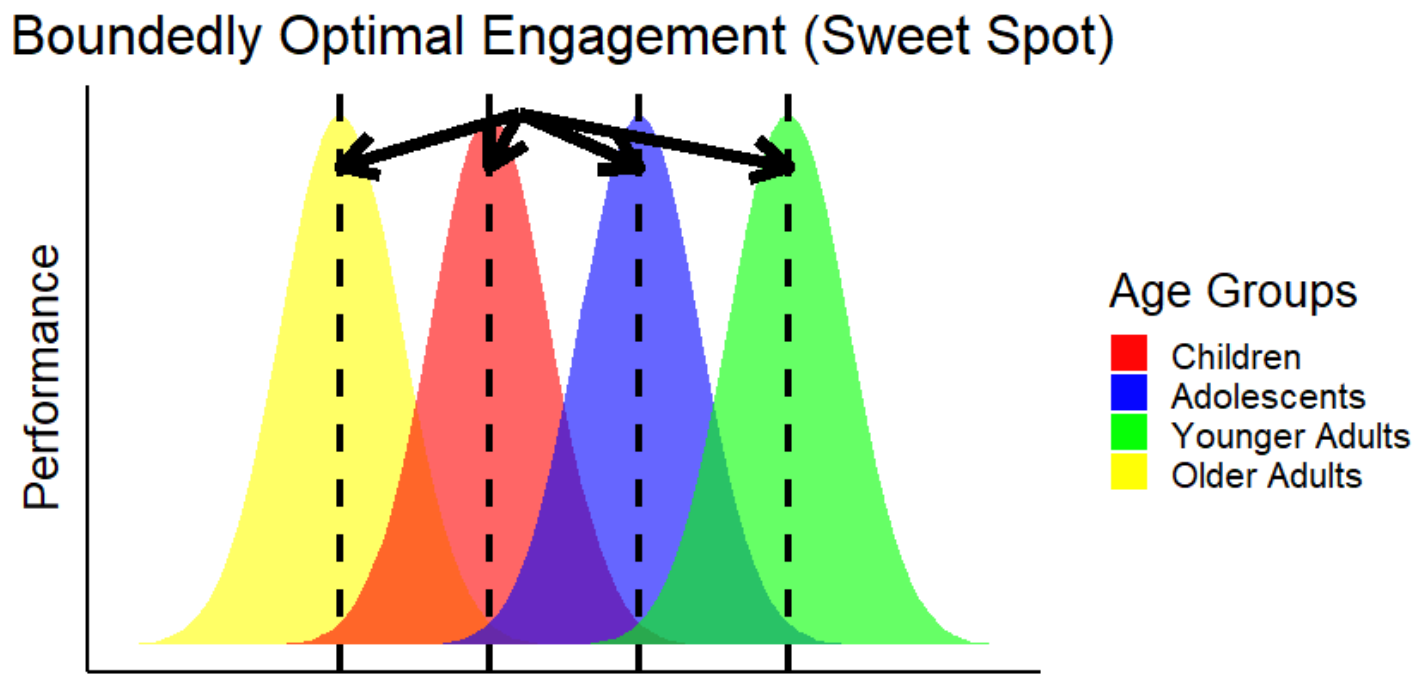

\section{Cognitive Abilities}

Fig 3. Visual representation of the sweet spot hypothesis. Effort engagement according to demands in cognitive control for children, adolescents, younger and older adults. Dashed lines represent the sweet spot for cognitive control for each age group.

Current theoretical models on motivation and cognitive control explain the behavior seen in children and older adults as a consequence of deficits in the ability to engage in meta-control and thus interpret their behavior as suboptimal (Dixon \& Christoff, 2012; Shenhav et al., 2013). In contrast, the two models we propose suggest that children and older adults are behaving optimally given the internal and external constraints that they face. 
Meta-control across the lifespan

Combining both the satisficing model as well as the sweet spot hypothesis may clarify how lifespan changes in cognitive control explain changes in meta-control. That is, individuals make optimal metacognitive decisions only when they are motivated to and have the available resources to do so (sweet spot hypothesis). Yet, when they fail on either of these criteria- to see additional value in effort investment for the relative computational costs the system must engage in-they will disengage in control (satisficing model). In this sense, we can see how the satisficing model and sweet spot models both reflect the core tenet of a resource-rational approach: namely that they consider optimal decision-making to be the best possible use of one's limited cognitive resources. Combining these models under a resource-rational framework highlights the idea that children and older adults are not suboptimal decision makers. Instead, their decisions are at least as rational as young adults' decisions.

\section{Conclusion}

When faced with a complex goal-directed task (e.g., navigating to a restaurant in a new city), we can apply different types of strategies such as using a GPS or learning the layout of the city. As we can engage in either strategy, we are faced with a meta-level problem; deciding which strategy to engage in. To solve this problem, individuals have been shown to engage in a cost-benefit analysis, weighing the benefits of the expected reward against the costs of engaging in each strategy. In the current paper, we reviewed how lifespan changes in cognitive abilities and motivational factors such as monetary incentives affect the engagement in these cost-benefit trade-offs. These findings suggest that children and older adults may be optimal decision-makers when considering the constraints of their cognitive abilities. As an extension of recent theories in young adults (e.g., reward-based models) (Shenhav et al., 2013), we propose two theoretical accounts based in resource-rationality, which integrate the rational use of limited resources with 
Meta-control across the lifespan

realistic cognitive constraints. Our theoretical accounts build on this framework by factoring in the idea of bounded rationality across the lifespan. Based on these models, we posit that children and older adults engage in optimal meta-control, trading off reward and effort, but that such control is bounded by structural and functional cognitive limitations. 
Meta-control across the lifespan

\section{References}

Beierholm, U., Guitart-Masip, M., Economides, M., Chowdhury, R., Düzel, E., Dolan, R., \& Dayan, P. (2013). Dopamine modulates reward-related vigor. Neuropsychopharmacology, 38(8), 1495-1503.

Bolenz, F., \& Eppinger, B. (2020, July 29). Valence bias in metacontrol of decision making in adolescents and young adults. PsyArXiv.

Bolenz, F., Kool, W., Reiter, A. M., \& Eppinger, B. (2019). Metacontrol of decision-making strategies in human aging. Elife, 8, e49154.

Botvinick, M., \& Braver, T. (2015). Motivation and cognitive control: from behavior to neural mechanism. Annual review of psychology, 66(1), 83-113.

Botvinick, M. M. (2007). Conflict monitoring and decision making: reconciling two perspectives on anterior cingulate function. Cognitive, Affective, \& Behavioral Neuroscience, 7(4), 356366.

Bruckner, R., Nassar, M. R., Li, S. C., \& Eppinger, B. (2020). Default beliefs guide learning under uncertainty in children and older adults.

Cattell, R. B. (1987). Intelligence: Its structure, growth and action. Elsevier.

Chevalier, N. (2018). Willing to think hard? The subjective value of cognitive effort in children. Child development, 89(4), 1283-1295.

Daw, N. D., Gershman, S. J., Seymour, B., Dayan, P., \& Dolan, R. J. (2011). Model-based influences on humans' choices and striatal prediction errors. Neuron, 69(6), 1204-1215.

Decker, J. H., Otto, A. R., Daw, N. D., \& Hartley, C. A. (2016). From creatures of habit to goaldirected learners: Tracking the developmental emergence of model-based reinforcement learning. Psychological science, 27(6), 848-858.

Devine, S. T., Otto, R., Bolenz, F., Reiter, A. M., \& Eppinger, B. (2019, December 9). Cognitive resource limitations shift effort trade-offs across the lifespan. PsyArXiv. 
Meta-control across the lifespan

Devine, S., Neumann, C., Otto, R., Bolenz, F., Reiter, A. M. \& Eppinger, B. (2020) Seizing the Opportunity: Lifespan Age Differences in the Effects of the opportunity Cost of Time on Cognitive Control. Manuscript submitted for publication.

Dixon, M. L., \& Christoff, K. (2012). The decision to engage cognitive control is driven by expected reward-value: neural and behavioral evidence. PloS one, 7(12), e51637.

Eppinger, B., Kray, J., Mecklinger, A., \& John, O. (2007). Age differences in task switching and response monitoring: Evidence from ERPs. Biological Psychology, 75(1), 52-67.

Eriksen, B. A., \& Eriksen, C. W. (1974). Effects of noise letters upon the identification of a target letter in a nonsearch task. Perception \& psychophysics, 16(1), 143-149.

Gershman, S. J. (2020). Origin of perseveration in the trade-off between reward and complexity. Cognition, 204(104394).

Griffiths, T. L., Lieder, F., \& Goodman, N. D. (2015). Rational use of cognitive resources: Levels of analysis between the computational and the algorithmic. Topics in cognitive science, $7(2)$, 217-229.

Hadley, L. V., Acluche, F., \& Chevalier, N. (2020). Encouraging performance monitoring promotes proactive control in children. Developmental Science, 23(1), e12861.

Hess, T. M., Smith, B. T., \& Sharifian, N. (2016). Aging and effort expenditure: The impact of subjective perceptions of task demands. Psychology and Aging, 31(7), 653.

Hsieh, S., Li, T. H., \& Tsai, L. L. (2010). Impact of monetary incentives on cognitive performance and error monitoring following sleep deprivation. Sleep, 33(4), 499-507.

Insel, C., Kastman, E. K., Glenn, C. R., \& Somerville, L. H. (2017). Development of corticostriatal connectivity constrains goal-directed behavior during adolescence. Nature Communications, 8(1), 1-10.

Kail, R., \& Salthouse, T. A. (1994). Processing speed as a mental capacity. Acta psychologica, 86(2-3), 199-225.

Kool, W., \& Botvinick, M. (2014). A labor/leisure tradeoff in cognitive control. Journal of experimental psychology: General, 143(1), 131-141. 
Meta-control across the lifespan

Kool, W., McGuire, J. T., Rosen, Z. B., \& Botvinick, M. M. (2010). Decision making and the avoidance of cognitive demand. Journal of Experimental Psychology: General, 139(4), 665682.

Kool, W., Gershman, S. J., \& Cushman, F. A. (2017). Cost-benefit arbitration between multiple reinforcement-learning systems. Psychological science, 28(9), 1321-1333.

Kool, W., Cushman, F. A., \& Gershman, S. J. (2018). Competition and cooperation between multiple reinforcement learning systems. In R. Morris, A. Bornstein \& A. Shenav (Eds.), Goal-directed decision making (pp. 153-178). Academic Press.

Kool, W., Shenhav, A., \& Botvinick, M. M. (2017). Cognitive control as cost-benefit decision making. In T. Egner (Ed.), The Wiley handbook of cognitive control (p. 167-189). Wiley Blackwell.

Kray, J., Eber, J., \& Lindenberger, U. (2004). Age differences in executive functioning across the lifespan: The role of verbalization in task preparation. Acta Psychologica, 115(2-3), 143-165.

Kurzban, R., Duckworth, A., Kable, J. W., \& Myers, J. (2013). An opportunity cost model of subjective effort and task performance. Behavioral and brain sciences, 36(6), 661-679.

Leotti, L. A., \& Wager, T. D. (2010). Motivational influences on response inhibition measures. Journal of Experimental Psychology: Human Perception and Performance, 36(2), $430-447$.

Lieder, F., \& Griffiths, T. L. (2020). Resource-rational analysis: understanding human cognition as the optimal use of limited computational resources. Behavioral and Brain Sciences, 43(E1).

Li, S. C., Hämmerer, D., Müller, V., Hommel, B., \& Lindenberger, U. (2009). Lifespan development of stimulus-response conflict cost: Similarities and differences between maturation and senescence. Psychological Research PRPF, 73(6), 777-785.

Li, S. C., Papenberg, G., Nagel, I. E., Preuschhof, C., Schröder, J., Nietfeld, W., ... \& Bäckman, L. (2013). Aging magnifies the effects of dopamine transporter and D2 receptor genes on backward serial memory. Neurobiology of aging, 34(1), 358-e1-358-e10.

Libby, R., \& Lipe, M. G. (1992). Incentives, effort, and the cognitive processes involved in accounting-related judgments. Journal of Accounting Research, 30(2), 249-273. 
Meta-control across the lifespan

Mayr, U., Spieler, D. H., \& Kliegl, R. (2001). Ageing and executive control: Introduction to this special issue. European Journal of Cognitive Psychology, 13(1/2), 1-4.

McDowd, J. M., \& Shaw, R. J. (2000). Attention and aging: A functional perspective. In F. I. M. Craik \& T. A. Salthouse (Eds.), The handbook of aging and cognition (p. 221-292). Lawrence Erlbaum Associates Publishers.

Menon, V., Boyett-Anderson, J. M., \& Reiss, A. L. (2005). Maturation of medial temporal lobe response and connectivity during memory encoding. Cognitive Brain Research, 25(1), 379385.

Nassar, M. R., Bruckner, R., Gold, J. I., Li, S. C., Heekeren, H. R., \& Eppinger, B. (2016). Age differences in learning emerge from an insufficient representation of uncertainty in older adults. Nature Communications, 7(1), 1-13.

Niebaum, J. C., Chevalier, N., Guild, R. M., \& Munakata, Y. (2019). Adaptive control and the avoidance of cognitive control demands across development. Neuropsychologia, 123, 152158.

Niv, Y., Daw, N. D., Joel, D., \& Dayan, P. (2007). Tonic dopamine: opportunity costs and the control of response vigor. Psychopharmacology, 191(3), 507-520.

O'Leary, A., \& Sloutsky, V. M. (2017). Five-Year-Old Children Transfer a Metacognitive Strategy to a Novel Task. Cognitive Science.

Ofen, N., Chai, X. J., Schuil, K. D., Whitfield-Gabrieli, S., \& Gabrieli, J. D. (2012). The development of brain systems associated with successful memory retrieval of scenes. Journal of Neuroscience, 32(29), 10012-10020.

Otto, A. R., \& Daw, N. D. (2019). The opportunity cost of time modulates cognitive effort. Neuropsychologia, 123, 92-105.

Padmala, S., \& Pessoa, L. (2011). Reward reduces conflict by enhancing attentional control and biasing visual cortical processing. Journal of cognitive neuroscience, 23(11), 3419-3432.

Patzelt, E., Kool, E., \& Gershman, S. J. (2019, February 17). Boosting model-based control in older adults. PsyArXiv. 
Meta-control across the lifespan

Ruel, A., Bolenz, F., Li S-C. \& Eppinger, B. (2020). Age differences in model-based decisionmaking under varying demands on the representation of state spaces. Manuscript in preparation.

Salthouse, T. A. (1990). Working memory as a processing resource in cognitive aging. Developmental review, 10(1), 101-124.

Shenhav, A., Botvinick, M. M., \& Cohen, J. D. (2013). The expected value of control: an integrative theory of anterior cingulate cortex function. Neuron, 79(2), 217-240.

Shenhav, A., Musslick, S., Lieder, F., Kool, W., Griffiths, T. L., Cohen, J. D., \& Botvinick, M. M. (2017). Toward a rational and mechanistic account of mental effort. Annual review of neuroscience, 40, 99-124.

Shohamy, D., \& Turk-Browne, N. B. (2013). Mechanisms for widespread hippocampal involvement in cognition. Journal of Experimental Psychology: General, 142(4), 1159-1170.

Simon, H. A. (1955). A behavioral model of rational choice. The quarterly journal of economics, 69(1), 99-118.

Simon, H. A. (1956). Rational choice and the structure of the environment. Psychological review, 63(2), 129-138.

Smid, C. R., Kool, W., Hauser, T. U., \& Steinbeis, N. (2020, January 24). Model-based decisionmaking and its metacontrol in childhood. PsyArXiv.

Starns, J. J., \& Ratcliff, R. (2010). The effects of aging on the speed-accuracy compromise: Boundary optimality in the diffusion model. Psychology and aging, 25(2), 377-390.

Strang, N. M., \& Pollak, S. D. (2014). Developmental continuity in reward-related enhancement of cognitive control. Developmental Cognitive Neuroscience, 10, 34-43.

Vassena, E., Silvetti, M., Boehler, C. N., Achten, E., Fias, W., \& Verguts, T. (2014). Overlapping neural systems represent cognitive effort and reward anticipation. PLoS One, 9(3), e91008.

Westbrook, A., Kester, D., \& Braver, T. S. (2013). What is the subjective cost of cognitive effort? Load, trait, and aging effects revealed by economic preference. PloS one, 8(7), e68210. 
Meta-control across the lifespan

Willis, S. L., \& Marsiske, M. (1991) Life span perspective on practical intelligence. In D. E. Tupper \& K. D. Cicerone (Eds.), The neuropsychology of everyday life: Issues in development and rehabilitation, pp. 183-197. Springer.

Yee, D. M., Adams, S., Beck, A., \& Braver, T. S. (2019). Age-Related differences in motivational integration and cognitive control. Cognitive, Affective, \& Behavioral Neuroscience, 19(3), $692-714$.

Zeithamova, D., Schlichting, M. L., \& Preston, A. R. (2012). The hippocampus and inferential reasoning: building memories to navigate future decisions. Frontiers in human neuroscience, 6(70) 1-14. 